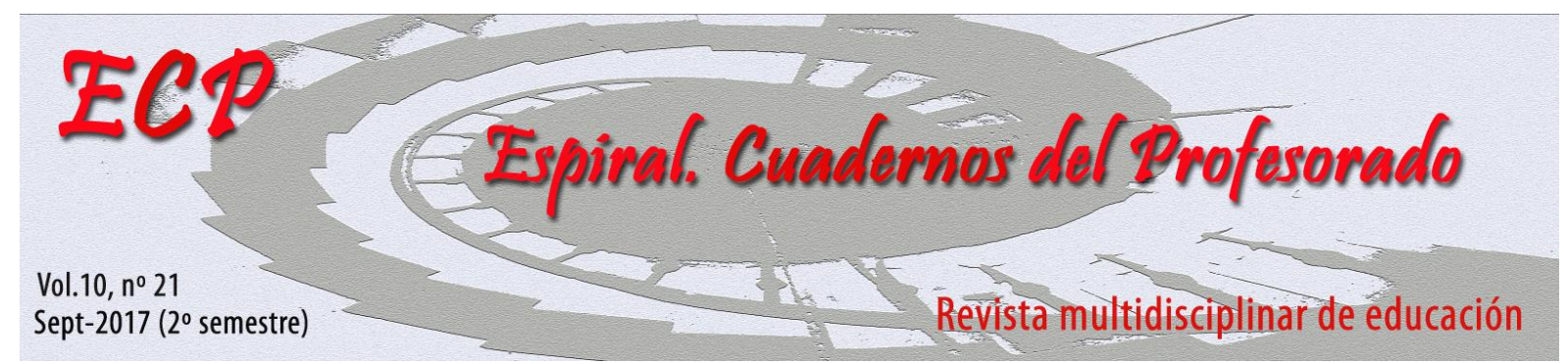

\title{
Situación actual de la formación en actividades en el medio natural según el profesorado de Educación Física. Un estudio cualitativo con expertos en Andalucía
}

\section{Current situation of the teacher training in outdoors activities by physical education teachers. A qualitative study with experts in Andalusia}

\author{
Jesús Sáez-Padilla, Inmaculada Tornero Quiñones, y Ángela Sierra Robles
}

Departamento de Didácticas Integradas, Universidad de Huelva, España

\begin{abstract}
RESUMEN: El objetivo de este estudio fue analizar la situación actual de la formación en actividades en el medio natural según el profesorado de Educación Física. Los datos de la investigación procedieron de entrevistas semiestructuradas realizadas a nueve docentes expertos en la formación del profesorado. Los resultados del estudio indicaron que la formación es escasa e insuficiente, tanto en la formación inicial como en la permanente. Sin embargo, se apostó por incrementar su desarrollo y mejora con fórmulas y formatos como la educación al aire libre, con sus salidas y estancias en plena naturaleza. Éstas nos permitieron abordar los diferentes contenidos curriculares de manera más activa. El pilar de esta mejora se basó en el aprendizaje experiencial que hace que el alumnado y profesorado estén mucho más implicados en el proceso de enseñanza-aprendizaje. La información ofrecida en este artículo puede ser de interés para establecer estrategias que favorezcan la mejora de la formación e investigación en el bloque de contenido actividades en el medio natural.
\end{abstract}

Palabras clave: formación del profesorado, formación inicial y permanente, currículum, actividades en la naturaleza, Educación Física.

\begin{abstract}
The aim of this study was to analyze the current situation of training outdoor activities as Physical Education teachers. The research data came from semi-structured interviews with nine experts training teachers. The results of this study indicated that training is limited and insufficient. However, it was decided to increase its development with formats such as outdoor education, with their stays in nature. These allow us to address the different curricula more actively. The cornerstone of this improvement is based on experiential learning that makes the students and teachers are more involved in the teaching-learning process. The information provided in this article may be of interest to develop strategies that promote improved training and research the outdoor activities.
\end{abstract}

Key words: teacher training, pre-service and in-service training, curriculum, outdoor activities, physical education.

Sáez Padilla, J., Tornero Quiñones, I., y Sierra Robles, A. (2017). La situación actual de la formación en actividades en el medio natural según el profesorado de Educación Física. Un estudio cualitativo con expertos en Andalucía. Espiral. Cuadernos del Profesorado, 10(21), 100-117.

Fecha de recepción: 19/04/2017

Fecha de aceptación: 19/07/2017
Ccorrespondencia:

jesus.saez@dempc.uhu.es (J. Sáez-Padilla) 


\section{Introducción}

Si los cambios en el sistema educativo pueden ser beneficiosos para toda la sociedad, no menos importante pueden ser las actuaciones que podamos tener en relación con la formación del profesorado en general, y de los docentes de Educación Física en particular. En nuestro caso hemos profundizado en un apartado concreto de la formación del profesor de Educación Física, las actividades en el medio natural como bloque de contenido a desarrollar como docente de Secundaria. Las salidas profesionales del Graduado en Ciencias de la Actividad Física y del Deporte han evolucionado acorde con las demandas de la sociedad, aumentan de forma exponencial las vinculadas a centros deportivos, gestión deportiva, entrenamiento, salud y actividad física, animación, recreación y por qué no, actividades en la naturaleza. Ya es un hecho la oferta tan amplia de empresas turísticas y deportivas cuyo soporte principal son las actividades al aire libre.

En este sentido Rivera (2010) considera que el medio natural ha experimentado un gran auge con diferentes modalidades deportivas en plena naturaleza, favoreciendo el disfrute del tiempo libre de manera activa a la vez que se disfruta del medio. En nuestro caso, nos interesa abordar un estudio donde las actividades en el medio natural sean el eje central por varios motivos Sáez-Padilla (2010): el medio natural como espacio educativo y formativo, las posibilidades educativas que ofrece este bloque de contenidos, las mejoras existentes en programas aplicados en otros países, apostar por propuestas innovadoras sin un gran desarrollo en nuestro país, vinculación directa con la educación en valores, el medio ambiente, el desarrollo personal o la salud.

Estamos convencidos que el medio natural como espacio educativo tiene un enorme potencial. Autores contrastados como (Rousseau, 1969) y su obra "El Emilio" así lo confirman, más recientemente, Richard Louv (2016) propone y justifica las posibilidades que tiene la naturaleza para una mejora a nivel global de la sociedad actual, aspectos relacionados con la salud y la educación son sus pilares. El medio se convierte en una gran aula, en la que podemos aprender y obtener mucha información. Para Mellado y Mellado (2006) los campamentos son experiencias educativas de primer orden. Sin embargo, como apunta Lourenço (2002) existe la confusión entre vida en la naturaleza con campamento, son formatos no incluidos en las instituciones educativas, pero estamos viendo que aportan muchas cosas positivas a la educación en general (Louv, 2016). En la actualidad contamos con múltiples inconvenientes, a solventar a medio y largo plazo, como iremos viendo con las propuestas de compañeros e investigadores. La realidad es la falta de recursos e infraestructuras para un desarrollo adecuado, así como la formación e implicación del profesorado. La superación de las dificultades permitirá que la vida en la naturaleza no se convierta en un mero campamento aislado del contexto educativo.

Existen trabajos como el de Parra (2001) que aporta datos sobre las principales causas para no incluir en el trabajo diario las actividades en el medio natural, configurando un primer paso para poder afrontar propuestas de mejora en un futuro. Algunas de las razones aportadas tienen que ver con el profesorado y la formación, destacamos: la falta de formación inicial del profesorado, la falta de ilusión docente, innovación e investigación, demasiado tiempo de preparación y puesta en práctica respecto a otras materias, un necesario aprendizaje de técnicas complejas y uso de material especializado y falta de conciencia política y social en relación a las actividades al aire libre.

Sin embargo, encontramos como en la actualidad las escuelas "Outward Bound" ofrecen una pedagogía de aventura intensa y corta, siendo el espacio formativo el medio natural. Por ejemplo, excursiones en la montaña y el mar junto con proyectos sociales, ecológicos, creativos y musicales (Nasser, 1994; Anglada, 2008). Una educación basada en la aventura permitirá (Nasser, 1994) el aumento de la motivación por el aprendizaje, así como la creación de objetivos educativos de forma experimental y entendible.

Este proceso es el propuesto por algunos autores como (Gómez Encinas, 2006; Parra, 2006; Santos \& Muñoz, 2007). En palabras de Granero y Baena (2007), cuando se lleva a cabo una unidad didáctica de actividades en el medio natural, resulta más fácil abordar la formación integral del alumnado, tan necesaria en estas edades, sobre todo, cuando se valora correctamente su potencial educativo. 
El trabajo a desarrollar con el profesorado pasa inexorablemente por una fase formativa que amplíe y mejore la formación actual. Algunas orientaciones de Santos (2003) muestran el camino a seguir, como la mejora de la formación inicial, el diseño de monográficos específicos, seminarios o proyectos de innovación. La aplicación de estos contenidos en la escuela y la innovación desde la práctica deben ser las bases de nuestras propuestas. Parra, Rovira y Luque (2002) opinan que este contenido se genera en un espacio formado por necesidades, demandas, novedad, validación pedagógica e innovación, permitiendo de esta manera afrontar estudios e investigaciones que puedan mejorar la situación actual, en la línea de autores citados anteriormente.

Por ello, el objetivo de esta investigación, llevada a cabo con profesores en activo y expertos en actividades en la naturaleza de la región andaluza, fue analizar la formación del profesorado de Educación Física en relación al bloque de contenido de actividades en el medio natural del currículum de Educación Física. La información obtenida en este estudio puede ser de utilidad para identificar aspectos que pueden ser susceptibles de mejora en el ámbito de la formación del profesorado. También nos servirá para diseñar estrategias destinadas a la mejora de este bloque de contenido en la Educación Secundaria Obligatoria.

\section{Método}

\section{Participantes y procedimiento de recogida de datos}

El universo de referencia está constituido por profesores expertos en actividades en el medio natural que imparten estas materias en el ámbito universitario y por profesores que han desarrollado una continua formación permanente especializándose en el contenido objeto de estudio e imparten clases en Educación Secundaria Obligatoria y en los ciclos formativos de la familia actividad física y deportiva. De los nueve sujetos (ocho son chicos y una chica), cuatro son profesorado de universidad, tres de ciclos formativos y dos de educación secundaria obligatoria. Nos encontramos ante un tipo de muestra no probabilística en la que se selecciona a los participantes haciendo énfasis en los casos más representativos y paradigmáticos y explotando a los informantes clave por la capacidad para obtener información relevante (Martínez, 2004). De esta forma se pretende que bajo el criterio del investigador se permita tener una muestra lo más representativa posible a efectos de la investigación.

Tabla 1.

Requisitos cumplidos por el profesorado entrevistado.

\begin{tabular}{cccccc}
\hline \multirow{2}{*}{ Entrevistado } & \multicolumn{5}{c}{ Requisitos } \\
\cline { 2 - 6 } & a & b & c & d & e \\
\hline E1 & $\mathrm{X}$ & $\mathrm{X}$ & $\mathrm{X}$ & $\mathrm{X}$ & $\mathrm{X}$ \\
E2 & $\mathrm{X}$ & $\mathrm{X}$ & $\mathrm{X}$ & $\mathrm{X}$ & $\mathrm{X}$ \\
E3 & $\mathrm{X}$ & $\mathrm{X}$ & $\mathrm{X}$ & $\mathrm{X}$ & $\mathrm{X}$ \\
E4 & $\mathrm{X}$ & $\mathrm{X}$ & $\mathrm{X}$ & $\mathrm{X}$ & $\mathrm{X}$ \\
E5 & $\mathrm{X}$ & $\mathrm{X}$ & $\mathrm{X}$ & $\mathrm{X}$ & $\mathrm{X}$ \\
E6 & $\mathrm{X}$ & & $\mathrm{X}$ & $\mathrm{X}$ & \\
E7 & $\mathrm{X}$ & $\mathrm{X}$ & $\mathrm{X}$ & $\mathrm{X}$ & \\
E8 & $\mathrm{X}$ & $\mathrm{X}$ & $\mathrm{X}$ & $\mathrm{X}$ & \\
E9 & $\mathrm{X}$ & & $\mathrm{X}$ & $\mathrm{X}$ & \\
\hline
\end{tabular}

Dos de las características de la muestra en esta etapa es que se realiza de forma intencionada y razonada. Se elige con criterios de representatividad del discurso, de tal forma que puedan responder a las preguntas de la investigación y que posibiliten conocer, descubrir e interpretar el fenómeno estudiado en profundidad, en sus diferentes visiones, de forma que refleje el problema con amplitud. 
El tipo de muestro es opinático (Fernández de Sanmamed, 2006). En el mismo, el poder de la muestra no depende del tamaño, sino de en qué medida las unidades de muestreo aportan una información rica, profunda y completa.

Los requisitos para la selección de los participantes $(n=9)$ son personas que cumplan al menos tres de las cinco características que se presentan (Tabla 1$)$.

a) Tener al menos cinco años de experiencia profesional docente relacionada con las actividades en el medio natural.

b) Publicaciones en revistas y libros sobre la formación del profesorado en actividades en el medio natural.

c) Diseño y desarrollo de proyectos de innovación y/o formación para la mejora de las actividades en el medio natural en el contexto educativo.

d) Miembro de un grupo de trabajo especializado en actividades en el medio natural.

e) Trabajar en líneas de investigación relacionadas con actividades en el medio natural.

La entrevista individual semi-estructurada es la técnica utilizada, con ello pretendemos, como opina Mogollón y Vázquez (2006) obtener información en profundidad sobre actitudes, valores y opiniones relativas al problema de investigación. El conocimiento de la realidad actual, así como sus necesidades y demandas, permitirá ofrecer mejoras y alternativas. Destacamos la labor formativa de los entrevistados en contenidos que son objeto de estudio, enriqueciendo de esta manera la información que podamos extraer de las entrevistas realizadas. Coincidimos con Cañadas, Ibáñez, García, Parejo, y Feu (2009), se tuvieron en cuenta las premisas de anonimato y confidencialidad de los datos, así como el consentimiento de los sujetos para participar en el estudio.

\section{Análisis de datos}

En todo proceso de análisis de datos cualitativos la secuencia base sigue las pautas recomendadas por Hernández, Fernández-Collado y Baptista (2006). En publicaciones recientes (Albarracín, Moreno, \& Beltrán, 2014) se llevó a cabo un estudio cualitativo coincidiendo con las pautas seguidas en nuestro trabajo. El proceso fue el siguiente: 1) grabar los datos con una grabadora y cintas SONY. 2) escuchar y escribir la información. 3) leer y organizar los datos, numerando, clasificando y delimitando oraciones. 4) analizar los contenidos y construir las dimensiones. Hay que identificar las dimensiones, buscar información relativa a cada categoría, identificar los diferentes aspectos relacionados con las dimensiones, escribir resúmenes e identificar ejemplos y citas. 5) Entrenamiento de los codificadores, expertos en la materia y la investigación cualitativa. 6) Codificar las entrevistas. 7) Utilización del programa informático AQUAD versión 5.8. 8) Describir los resultados en función de las semejanzas y las diferencias. 9) Finalmente interpretar los resultados siempre relacionado con el objeto de estudio.

Coincidiendo con Albarracín, Moreno, y Beltrán (2014) la participación de varios investigadores en el proceso mejoró la credibilidad de la investigación y redujo la influencia de sesgos personales, así como todo el proceso de recodificación y recategorización que dio soporte a la sección de resultados (Tabla 2).

Tabla 2.

Sistema de categorías y subcategorías tras el análisis de datos.

\begin{tabular}{lll}
\hline Categorías & Subcategorías & Códigos \\
\hline Valoración de la & Experiencias vividas en & Mucha teoría y poca práctica \\
\cline { 2 - 2 } $\begin{array}{l}\text { formación inicial en } \\
\text { actividades en el medio } \\
\text { natural por parte del } \\
\text { profesorado experto }\end{array}$ & & Pocas horas y créditos de formación \\
\cline { 2 - 2 } & & $\begin{array}{l}\text { Poca interacción entre la universidad y la realidad } \\
\text { educativa }\end{array}$ \\
\cline { 2 - 2 } & &
\end{tabular}

Falta de apoyo institucional para llevar a cabo esta 
formación

Análisis de la formación Incoherencia en los itinerarios formativos existentes recibida

Fallo en la conexión entre la teoría y la práctica

La necesidad de utilizar el medio natural como espacio educativo

Propuestas de mejora Creación de un formato común y básico en los planes de estudios

Incremento de asignaturas de medio natural en la formación inicial de universidades

Enfoque educativo para acercarlas al centro

La necesaria utilización de otros tiempos pedagógicos para una formación adecuada.

Valoración de la formación permanente en actividades en el medio natural por parte del profesorado experto
Análisis de la situación actual de la formación permanente

Demanda poco específica y escasa del profesorado

Poca oferta formativa

Propuestas de mejora Actualización relacionada con aspectos didácticos

Aplicación práctica de los contenidos

Ampliar la colaboración con otras instituciones

Contenidos básicos a impartir

Aportaciones del profesorado experto al perfil del docente a impartir formación en actividades en el medio natural del experto en natural
Contenidos de aire libre adaptados a contextos escolares y urbanos

Competencias básicas actividades en el medio

Adaptación de contenidos al aire libre al centro educativo y entornos urbanos

Dominio contenidos mínimos del currículo

Enfocar la enseñanza desde un enfoque multidisciplinar e integral

Capacidad para el trabajo colaborativo

Itinerario básico para el Profesorado de educación física en activo futuro docente

Tener experiencias básicas en actividades en el medio natural

Incrementar la colaboración centros educativos y universidad para garantizar innovación e investigación

Propuestas de mejora Mayor implicación del profesorado

Incentivar la formación del profesorado en activo

Buscar apoyo de instituciones y expertos en los contenidos a trabajar 


\section{Resultados}

En este apartado y tras el análisis de las entrevistas se exponen los resultados obtenidos. Partiendo de las categorías señaladas (Tabla 2) utilizamos fragmentos de las entrevistas que nos ayudan a corroborar los datos.

\section{Valoración de la formación inicial en actividades en el medio natural por parte del profesorado experto}

\section{Experiencias vividas en la formación inicial}

La mayoría del profesorado entrevistado declaró un interés muy alto por las actividades en el medio natural pero la formación recibida tenía muchos aspectos a mejorar.

De hecho, en cuarto de INEF participé como becaria del Departamento de Ocio y Recreación, donde pude cumplir un sueño, hacer la memoria de licenciatura sobre actividades en la naturaleza (E2).

Nos mostraron un escaparate de lo que había sin más. Nos habría hecho falta más tiempo lectivo y aplicación práctica de los contenidos teóricos (E3).

Cuando yo estudié hace más de 20 años, estaba poco sistematizado el bloque. Recuerdo con especial cariño las acampadas, las salidas a la nieve y las salidas a escalar. Creo que en aquellos entonces no se les podían pedir peras al olmo, estábamos en los albores del desarrollo de la propia profesión (E4).

En el campamento hice práctica de piragüismo y escalada con Fulgencio, pero no me formaron en estos contenidos. Nos mostraron un escaparate de lo que había sin más. Me habría hecho falta más tiempo lectivo. Otro contenido recibido fueron las técnicas de aire libre, por ejemplo, la cabuyería, pero me faltó la aplicación práctica. Me falto conocimientos sobre material y equipamientos. En la actualidad, la evolución de los materiales es tan rápida que tienes que actualizarte continuamente, en muchas disciplinas puedes quedar desfasado en uno cuantos meses. Considero que conocimientos sobre meteorología y astronomía también me faltaron. Actualmente son contenidos que utilizo mucho, concretamente la astronomía para mí es un complemento muy interesante (E9).

La realidad es que el profesorado entrevistado con mucha experiencia en el ámbito formativo consideró en términos generales que la formación ha sido escasa. Hizo hincapié en que la preparación entre la teoría de la facultad y la práctica en situaciones reales no es adecuada, quizás demasiado centrado en aspectos teóricos y casi inexistente en afrontar de forma coherente y con recursos suficientes el desarrollo de la práctica docente en el día a día en la escuela. Vemos algunos ejemplos:

Bien desde mi perspectiva, no tengo muy buenos recuerdos en cuanto a la formación recibida, no fue muy alta, incluso en cuarto de carrera no cogí la asignatura (E5).

Dentro del programa obligatorio se veían muchos contenidos, pero el problema era la carga lectiva, tres créditos y dos horas a la semana. Las asignaturas de naturaleza son escasas (E7).

Al tener solo una asignatura obligatoria y el resto optativas, al no ser una formación troncal y obligatoria, al ser el currículo tan amplio se acaba priorizando en otros bloques que no es el de actividades en el medio natural (E8).

Teóricamente sales con una preparación del INEF pero te das cuenta que no es los suficiente y que después a la hora de la verdad la realidad y la práctica son diferentes. Hombre la formación que tuvimos, además nuestra promoción por diversas causas, fue incluso más escasa de lo habitual (E9).

\section{Análisis de la formación recibida}

La formación inicial recibida ha sido muy básica e insuficiente en la mayoría de los casos. Aunque existían prácticas muy consolidadas como acampadas y campamentos no han permitido una 
consolidación de la formación básica en actividades en el medio natural. En el mejor de los casos tuvieron dos asignaturas en toda la carrera. En general eran muy pocas horas para muchos contenidos y además no estaban integradas en la troncalidad y obligatoriedad. Otro de los aspectos era la poca relación de la teoría con la práctica en situaciones reales. Se daban muchos aspectos teóricos y pocos prácticos con deficiencias en los recursos para llevar a cabo las prácticas. Algunos ejemplos:

Bien desde mi perspectiva, no tengo muy buenos recuerdos en cuanto a la formación recibida, no fue muy alta, incluso en $4^{o}$ de carrera no cogí la asignatura (E5).

Dentro del programa obligatorio se veían muchos contenidos, pero el problema era la carga lectiva, tres créditos y dos horas a la semana. Las asignaturas de naturaleza son escasas (E7).

Al tener solo una asignatura obligatoria y el resto optativas, al no ser una formación troncal y obligatoria, al ser el currículo tan amplio se acaba priorizando en otros bloques que no es el de actividades en el medio natural (E8).

Teóricamente sales con una preparación del INEF, pero te das cuenta que no es los suficiente y que después a la hora de la verdad la realidad y la práctica son diferentes. Hombre la formación que tuvimos, además nuestra promoción por diversas causas, fue incluso más escasa de lo habitual (E9). positivas:

A pesar de la deficiente formación inicial existían algunas experiencias formativas muy

Yo tuve lo que se hacía en $2^{\circ}$ de INEF con una asignatura cuatrimestral y en $4^{o}$ de INEF con otra asignatura cuatrimestral en la que se realizaba un campamento de 10 días donde realmente se llevaban a cabo todo este tipo de actividades (E3).

\section{Propuestas de mejora}

Entre las propuestas planteadas por los entrevistados destacamos la elaboración de un formato o itinerario común para todo el territorio nacional sin olvidar las características de cada zona o región. El diseño de una asignatura con el perfil de didáctica de las actividades en el medio natural para afrontar la variedad de contenidos y especialidades que se trabajan.

Los contenidos y actividades pueden variar dependiendo los lugares donde se desarrollen, es decir, el contexto educativo puede variar la actividad a desarrollar, pero el modelo permanecería. Haría falta el consenso del profesorado que imparte estas asignaturas en la Universidad para establecer unos criterios comunes (E1).

Crear a lo mejor algunas asignaturas en las que se trabaje eso a nivel de pedagogía, didáctica de las actividades en la naturaleza (E7).

Todo el profesorado entrevistado entiende que es necesario un cambio y modificación del sistema actual de la formación inicial en las universidades. Dadas las características del profesorado como la experiencia acumulada ofrecen algunas alternativas muy interesantes y a tener en cuenta en un futuro. Desde el incremento de las asignaturas en el itinerario formativo, pasando por un enfoque más educativo y no tan técnico (aunque es imprescindible este apartado) hasta la utilización de otros tiempos pedagógicos para su correcta formación en competencias básicas y necesarias.

Creo que se debería establecer un itinerario específico en estos temas, que funcionara si se quiere como una maestría, en los que se incluyeran temas sobre deportes de deslizamiento, aprovechamiento turístico, gestión y organización de actividades de estancia en la naturaleza, recreación y animación, salud, actividades y tercera edad, empresas... (E4).

Incremento del número de créditos en estas materias en la formación inicial, permitir la posibilidad de especialización deportiva y recreativa en este campo (E4).

Vamos a ver, yo soy bastante afortunado porque el profesor que impartía esa clase, abrió un abanico muy amplio y ofertaba no solamente la asignatura con el 
programa oficial de la asignatura de actividades en la naturaleza, sino que, a la vez, también organizaba cursos voluntarios en los que podíamos participar (cursos de orientación, escalada, bicicleta de montaña, supervivencia...) vinculados a la asignatura (E7).

Creo que la asignatura debería haber sido anual y haber recibido una formación para adaptar las actividades el aire libre al entorno urbano y escolar. Contenidos de Educación Ambiental, yo no recibí nada, nadie me lo contó. No debemos ser biólogos, pero si nos deben dar un prisma para un desarrollo sostenible. También una metodología y didáctica de las actividades al aire libre. Como coordino y controlo a 40 personas en el medio natural. Por otro lado, echo de menos la experiencia directa, o sea, la práctica (E9).

\section{Valoración de la formación permanente en actividades en el medio natural por parte del profesorado experto}

\section{Análisis de la situación actual de la formación permanente}

Las principales aportaciones fueron mínima formación del profesorado en activo, la escasa demanda de formación específica por parte del mismo y la escasa oferta formativa ofertada por los responsables. Son formatos muy generalistas, que, con sus ventajas, son mayores los inconvenientes, que pueden ser superados por la implicación del profesorado.

La mayoría no tienen formación, pueden tener conocimientos, pero no tienen seguridad pues carecen sobre todo de la práctica. En la actualidad, la evolución de los materiales es tan rápida que tienes que actualizarte continuamente, en muchas disciplinas puedes quedar desfasado en uno cuantos meses (E9).

En el caso de los docentes, la mayoría está asistiendo a actividades del Centro del Profesorado, pero el problema es que, en ocasiones, los accesos a la formación no sólo son del área de Educación Física, sino también de otras áreas, y por lo tanto no pueden priorizar un curso de específico de E.F. (E8).

La mayoría no tienen formación, pueden tener conocimientos, pero no tienen seguridad pues carecen sobre todo de la práctica. En la actualidad, la evolución de los materiales es tan rápida que tienes que actualizarte continuamente, en muchas disciplinas puedes quedar desfasado en uno cuantos meses (E9).

\section{Análisis de los formatos actuales de formación y propuestas de mejora}

De manera casi unánime la respuesta fue el seminario de formación permanente con la implicación de los centros educativos, el profesorado y la universidad. Acuñando además la idea de involucrar a ayuntamientos, federaciones y demás instituciones. Y contar, si fuera necesario, con el apoyo externo de especialistas para completar la formación. Entre las mejores opciones la actividad formativa con las siguientes características: un $70 \%$ de práctica y un $30 \%$ de teoría a realizar tanto en el centro como en los entornos próximos. Esta formación podría pasar por dos fases, la primera a modo de recetas y la segunda con una formación centrada en los fundamentos y adaptaciones a los contextos escolares y urbanos. La unidad didáctica nos serviría como modelo contextualizado, en definitiva, debemos ofrecer al profesorado propuestas formativas atractivas y prácticas.

Destacar la vigencia de un seminario de formación permanente donde intentamos llevar a la práctica estos contenidos en colaboración con los colegios y profesorado que se implica en llevarlos a cabo (E1). El formato más adecuado sería un seminario permanente de trabajo con aplicación a la realidad educativa, más que máster o curso de expertos. Aunque tampoco lo tengo muy claro. Desde la Universidad y en colaboración con otras entidades como federaciones, ayuntamientos, centros del profesorado... (E2). Optaría por una formación continua, bajo el formato de Seminario Permanente, lo que permite hacer frente a los continuos cambios que se producen en el área (E9). 
Otras de las fórmulas planteadas es que el formato puede variar dependiendo del número de profesores, así un departamento con un número elevado (centros con ESO, Bachillerato y Ciclos Formativos) podría solicitar una actividad formativa para ese departamento, si no es posible, pasaríamos al formato tradicional a nivel local, provincial o incluso regional. Sin olvidar los cursos de expertos y los cursos monográficos con una tipología diferente, así como la duración. Éstos servirían de base para la formación de grupos de trabajos que desarrollen proyectos comunes, que se experimentan las propuestas diseñadas, que haya una puesta en común posterior... También desde la universidad podrían celebrarse actividades formativas para los egresados, que sirvieran de actualización en todos los aspectos.

Cursos de expertos por si te quieres especializar en una materia concreta y, CEP que fueran más generalistas tanto para maestros como licenciados, ciclos formativos... (E5).

En un centro con un entorno idóneo para actividades de media montaña, alta montaña y actividades acuáticas de cualquier índole. Podrían organizarse cursos solamente para el profesorado de un solo centro (cuando sea numeroso, a partir de cuatro profesores), o pasaríamos a un formato de ámbito local, provincial o regional (E6).

Pero sobre todo que la formación continua sea aplicable, que no sea utópico lo que se plantea. Pues cursos monográficos con una tipología de distinta duración y que esos cursos sirvan para avanzar y el otro formato que es los grupos de trabajo y seminarios, y el enseñar a la gente a que el trabajo en grupo es el que funciona y que una vez que nos han dado un curso, por ejemplo sobre la bicicleta de montaña, dentro del ámbito educativo que luego se genere, se propicien desde los CEP en generar grupos de trabajos, seminarios para que luego lo que nos han contado en el curso lo pongamos en práctica unos cuantos profesores y veamos: esto funciona, esto no funciona, esto se puede modificar de esta manera ... (E7).

El formato sería a través del CEP o la Universidad, en un camino rápido, así como la calidad de los contenidos porque los ponentes y sus aportaciones suelen tener un reconocido prestigio. La forma idónea tendría que ser: fin de semana, 2 días, acampada... (E9).

Finalmente, entre otras mejoras apuntaron reforzar los vínculos y de manera real entre centros educativos, centros de formación del profesorado y la universidad. En segundo lugar, el enfoque de la formación permanente debe ir encaminado a llenar las lagunas formativas del profesorado, así como su aplicación en el ámbito educativo, pero desde un enfoque multidisciplinar.

Creo que esta formación tiene que ir todo unido, importante desde los centros educativos, desde los CEP, pero siempre en línea con la universidad, porque la universidad es donde se está generando es tipo de formación, aquí la investigación es muy importante (E7).

Cada docente debe asumir aquellos contenidos para los que se encuentra capacitado. A partir de aquí acudimos a resolver otro de los grandes inconvenientes que la aplicación de las actividades en el medio natural en el ámbito educativo (E7).

Si nos centramos en la formación del profesorado, el tratamiento de los contenidos no solo a nivel técnico, sino a otros niveles, a nivel de sensaciones y educativo, el cómo la escalada puede influir a nivel educativo, que valores puede trasmitir a los chavales (E7).

\section{Contenidos básicos a impartir}

En los apartados anteriores hemos visto que la formación inicial es deficitaria, tanto en contenidos como en carga lectiva, y por otro lado nos encontramos con la diversidad de contenidos que podemos abordar a nivel formativo. Las actividades en el medio natural aglutinan muchas disciplinas o materias, y aunque algunas, su enseñanza no corresponde al instituto, por razones obvias 
(algunos ejemplos como submarinismo, paracaidismo...), si es verdad, que nos encontramos en la tesitura de elegir que contenidos debemos enseñar. Para ello, los expertos entrevistados aportaron algunas orientaciones para mejorar la situación actual. Veamos algunos ejemplos:

Los contenidos que se trabajen que sean contenidos reales, contenidos aplicables al aula, es decir, en un curso de escalada, realizas la práctica en la roca, escalas de primero, no nos vale, debemos ofrecer contenidos que se puedan llevar a cabo en el aula (E7).

Considero que sería interesante contenidos relacionados con actividades en el medio acuático, dependiendo de la zona geográfica donde se impartan las clases, relacionadas con el medio natural. También sería interesante hablar de primeros auxilios y accidentes en el medio natural y su puesta en práctica real en lo que pueda ocurrir y la importancia de contenidos relacionados con la responsabilidad civil docente en sus salidas al medio natural (E5).

Todos aquellos de aire libre que se pueden adaptar a los contextos escolares y urbanos (E9).

\section{Aportaciones del profesorado experto al perfil del docente a impartir formación en actividades en el medio natural}

\section{Competencias básicas del experto en actividades en el medio natural}

A la pregunta ¿cómo sería el profesor ideal para impartir formación en contenidos de aire libre? el profesorado entrevistado coincidió en que debe ser un profesorado de educación física en activo con experiencia en estos contenidos y profesorado universitario experto en estas materias que aseguren investigación e innovación en el área. Aunque también explican cómo ciertos aspectos técnicos de algunos contenidos deben ser impartidos por técnicos especialistas con una buena formación didáctica que sepan conjugar muy bien la teoría y la práctica. Algunos ejemplos:

Tampoco creo en el técnico en escalada (por poner ejemplos) que imparte cursos de escalada deportiva para la educación secundaria sin tener experiencia en dicho ámbito (E1).

Dado el escaso desarrollo de nuestro bloque, podemos encontrar gente muy formada alejados de los caminos de la educación formal, si son buenos hay que saber que nos pueden contar. Pocos son los que realmente saben de todo. Los ideales serían los que conjugarán en su docencia aspectos educativos, técnicos y vivenciales (E4).

Dado el escaso desarrollo de nuestro bloque, podemos encontrar gente muy formada alejados de los caminos de la educación formal, si son buenos hay que saber que nos pueden contar. Pocos son los que realmente saben de todo. Los ideales serían los que conjugarán en su docencia aspectos educativos, técnicos y vivenciales (E4).

Profesorado cualificado (profesorado de enseñanza secundaria, profesorado de formación profesional de grado medio o superior, titulados por las federaciones deportivas, técnicos de los servicios deportivos de las universidades...), pero que conozca la problemática de los centros y del estado actual del alumnado (E6).

La tendencia sería buscar profesorado que supieran conjugar lo teórico y lo práctico. El perfil sería el de formador especialista en niveles adecuados. Hay que tener cuidado con el tema de los monitores, pues como no existen especialistas cualificados se están utilizando para desarrollar contenidos de este tipo (E9).

Una combinación de: profesores de educación física en activo, con experiencia en estos contenidos y profesorado universitario expertos en esta materia que aseguren investigación e innovación en el área (E10).

\section{Itinerario básico para el futuro docente}


El profesorado que imparta la formación de estos contenidos, debe ser un profesional que tenga experiencias en este campo, con una perspectiva integral, donde se conjuguen aspectos técnicos, didácticos, teóricos y prácticos. Debemos valorar la calidad del profesorado, independiente del ámbito formativo que proceda. Toda acción formativa debe estar contextualizada. En nuestro caso, el profesorado de educación física, las propuestas formativas deben ir encaminadas al trabajo de las actividades en el medio natural en los centros educativos, con un enfoque muy práctico, pero con unas bases teóricas, prácticas y didácticas sólidas. Se debe conjugar la formación reglada más integral y generalista con formación especializada debido a que un profesor de Educación Física no puede ser especialista en todo.

Es necesaria una formación donde se conjugue muy bien los aprendizajes técnicos con las posibilidades educativas que implica el contacto con el medio natural (E2).

La experiencia tuya le va a quedar mucho más claro, por lo tanto, el docente se lo tiene que creer, y como se lo tiene que creer debe estar familiarizado con el tema. Por ejemplo, si voy a hablar de la bicicleta, debo conocer tanto los aspectos teóricos como prácticos de la misma (E8).

En mi opinión, éste es el verdadero trabajo del profesorado, la contextualización del proyecto educativo (E1).

Por lo tanto, la formación del educador vendrá dada por la progresión lógica de aprendizajes desde los más generales (maestros) hasta los más técnicos (sin llegar a ser monitores de montaña en Secundaria (E2).

No creo que el profesor de Educación Física tenga que ser un monitor de ocio y tiempo libre y que tenga que saber de todo. Para solucionar esta situación, mantengo relación con la facultad del deporte, concretamente con las asignaturas de medio natural, en la que el alumnado formado en estas materias, participa, en el trabajo desarrollado en el centro educativo, como experto (E7).

Lo ideal, estaría compuesto por un equipo multidisciplinar, tanto médico que estuvieran relacionados con los temas de la montaña, salud, prevención de riesgos, lesiones típicas..., pero sobre todo expertos y especialistas formados en actividades en el medio natural a nivel oficial, aunque también podemos contar con el profesorado y los especialistas del ciclo formativo de grado medio. Pensamos que tienen mucho que aportar por su experiencia, motivación y por qué no solo quieren aprender, sino también trasmitir lo aprendido (E5).

\section{Propuestas de mejora}

Las posibles mejoras propuestas por los expertos, han sido: la formación inicial, modificando los planes de estudios e incrementando la presencia de estos contenidos. La implicación del profesorado en actividades formativas con formatos participativos como puede ser el trabajo colaborativo. Apoyo externo de expertos en contenidos específicos y respaldo institucional en todas las facetas en las que se desarrolla el hecho educativo: tiempos pedagógicos, salidas, centro, etc. Ejemplos:

Para afianzarlos en los centros educativos necesitamos profesionales implicados en el tema y esto se consigue con una buena formación inicial basada en la práctica y con apoyo institucional en toda regla, como ya hemos comentado (E1).

Más formación inicial y permanente, cultura de colaboración, alternativas para aplicar las actividades en el medio natural en medios más cercanos al centro, así como una información e implicación de la comunidad educativa (E2).

Pues sería unificar y controlar jurídicamente y administrativamente por ley, que el que impartiera u organice este tipo de actividades, estuviera lo más homologado posible, que estuvieran impartidas por especialistas (E5). 
Pues profesores expertos, pero no simplemente un experto machaca que haya subido ocho veces al Everest, debe plantear situaciones que se puedan aplicar en el aula, debemos unir las dos vías, lo técnico y lo didáctico. Al final lo importante de verdad es lo didáctico (E7).

Profesorado con un perfil diversificado tal como he ido argumentando anteriormente. Educador y técnico (E2).

\section{Discusión}

En este artículo se analiza la situación actual de las actividades en el medio natural desde una visión del profesorado de Educación Física que trabaja este bloque de contenido dentro del currículum de Educación Física en la Educación Secundaria Obligatoria de la región de Andalucía. En este apartado se discute y se identifican aspectos positivos o negativos que afectan a la enseñanza de la Educación Física en general y a las actividades en el medio natural en particular.

En cuanto a la primera categoría, la valoración que hace el profesorado experto sobre la formación inicial en actividades en el medio natural. Como aspecto básico el profesorado entrevistado destaca la influencia de las experiencias vividas tanto en la formación inicial como en su estilo de vida muy vinculado a las actividades al aire libre. Siedentop (1998) y más recientemente, Castillo y Almonacid (2012) y Albarracín, Moreno y Beltrán (2014) plantean como las experiencias positivas y una vocación por el trabajo en el campo de la actividad física permitirán probablemente un mejor formación y superación de los obstáculos actuales para una adaptación a las realidades actuales.

La formación inicial recibida ha sido escasa e insuficiente, así lo demuestran los planes de estudios actuales y la opinión de los entrevistados (Sáez-Padilla, 2010). Para Pereira y Félix (2002) las nuevas demandas sociales no son correspondidas con una formación adecuada en las instituciones universitarias, en la actualidad los planes formativos siguen teniendo poca carga lectiva con asignaturas relacionadas con la naturaleza (Sáez-Padilla, 2010; Sáez-Padilla y Fuentesal, 2014). El alumnado en su formación inicial no tiene experiencias motrices relacionadas con las actividades en el medio natural, unido en ocasiones a la falta de innovación e investigación en este campo además de falta de conciencia política y social con estos contenidos (Santos, 2003; Parra, 2001). La opinión de Higgins et al. (2014) nos parece muy acertada para incluirla en la situación actual de la formación inicial de las universidades, el problema de los costes, tiempo y seguridad sigue siendo un bache difícil de superar en las escuelas de primaria y secundaria.

Para atender las demandas de los profesionales se diseñan algunas propuestas para mejorar la situación actual. Sáez-Padilla y Fuentesal (2014) explican cómo la Universidad de Huelva apuesta por un itinerario específico en actividades al aire libre que cubre las necesidades actuales en cuanto a la formación inicial en los planes de estudios, con más de cinco asignaturas en esta etapa formativa.

Cuando nos enfrentamos al hecho educativo, sobre todo los primeros años, todas nuestras referencias las encontramos en la formación inicial. Por ello consideramos que ante la realidad educativa los cambios deben venir también desde la fuente del conocimiento como es la Universidad. Sin embargo, la realidad según Jaén y Banet (2003) es que el contexto y las características de los programas de formación inicial de los profesores de secundaria distan mucho de ser los adecuados para que los futuros docentes puedan afrontar, con ciertas garantías de éxito, la difícil empresa de enseñar al alumnado como aprender a aprender.

La búsqueda de experiencias y otros trabajos de investigación que mejoren la situación descrita hacen más fácil su incorporación al ámbito formativo y trabajo en los centros educativos. La propuesta planteada por Allain, Claquin, y Ollier (2005) en las escuelas de Francia, ayuda a comprender el enfoque que debe darse al trabajo de estos contenidos reconociéndoles dos vocaciones: la primera, en relación al medio natural, caracterizado por la incertidumbre de un medio cambiante, se demanda la adaptabilidad de las respuestas motrices, la anticipación y la gestión de los riesgos. La segunda consiste en favorecer que los alumnos encuentren prácticas sociales y culturales que una gran parte de ellos no descubrirían sin la escuela. En proyectos más actuales, las escuelas al aire libre de Noruega han sido incluidas en la educación formal, favoreciendo también la utilización del tiempo 
libre en contacto con naturaleza, el hecho de sentir placer al estar en la naturaleza va ligado con tomar acciones en protegerla, así como la oportunidad de aprender jugando, ser autónomos y descubrir las posibilidades del entorno olvidando su "hostilidad" y aprender a prepararse para afrontarlo y disfrutarlo (Sánchez, 2014).

Algunas de las mejoras propuestas tienen coincidencia con estudios anteriores (Napper-Owen et al., 1999; Salinas, 2006). Es necesario que desde la universidad y sobre todo su profesorado acepte la responsabilidad de preparar y concienciar al profesorado de Educación Física en su formación inicial, de cara a que éstos incorporen e incluyan en sus planificaciones actividades innovadoras.

Como comenta Vegas (2006), tenemos la necesidad de mejorar la calidad de la enseñanza. Para ello, los docentes son una de las piezas claves en el cómo y cuánto enseñar. Posiblemente estemos ante uno de los desafíos más importantes para la educación, el establecimiento de un conjunto de incentivos que garanticen la atracción, el no abandono y la motivación del profesorado ante las dificultades actuales de la profesión. Ya desde la formación inicial se deben establecer las bases de una formación de calidad centrada en el profesorado.

Como primer paso para el desarrollo profesional es necesario establecer mecanismos que faciliten el acceso a la problemática real de las aulas, los docentes y el alumnado desde el principio (García y Martínez, 2003). Para Pila Teleña (1981) lo primero que tiene que hacer un profesor es formarse y luego necesita actualizarse constantemente porque la tarea docente no es fácil. También debe convencerse a uno mismo, luego a los alumnos y a los padres, de que lo estás haciendo bien. Y esto sólo es posible desde el conocimiento profundo.

Como apuntan Fullonet y Fuster (2004) ya se ha pasado la época de realizar las actividades en el medio natural de forma puntual (fiestas, salidas, viajes o convivencias), es el momento de impulsar estas actividades dentro del centro, apostando por una educación transversal y de respeto hacia el medio ambiente. También corresponde a las instituciones con vínculos directos en formación cambiar para adaptarse a los nuevos tiempos. Acuñamos algunas de las propuestas que están dando resultados en otros países como es el outdoor education (educación al aire libre). En la Universidad de Edimburgo (Escocia), Higgins y Kirk (2002) apuestan por iniciativas formativas como la denominada: "Teacher Education, Outdoor Education and Sustainability in Scotland" en la que aparece la educación al aire libre como un bloque transversal.

La segunda categoría, la valoración del profesorado entrevistado sobre la formación permanente es que existe poca formación, los formatos son muy generalistas y poco adaptados a las actividades en la naturaleza. Se plantea también la necesidad de una actualización a los nuevos contenidos, así como una aplicación práctica de los nuevos y los más trabajados (Sáez-Padilla, 2010). En la actualidad las actividades formativas se ciñen a un horario convencional que da cabida a formatos poco abiertos, sin embargo, las nuevas tendencias nos llevan a otra lectura sobre el formato más utilizado. En las actividades al aire libre es necesario la utilización de formatos muy variados y, sobre todo, que se desarrollen en algún momento, en el medio natural. Estas premisas hacen que afrontemos propuestas formativas con diseños no convencionales pero necesarios (Arribas, 2008; Parra, 2001; Sáez-Padilla, 2010; Santos, 2003). Bacaicoa y Marín (2001) comentan que la práctica educativa como tal, no favorece la creación de procesos que promuevan la profundización en los principios teóricos y en las tesis básicas que deben iluminar la actividad profesional en el contexto escolar. Y cuando se llevan a cabo actividades formativas, el éxito de las mismas depende en gran medida de la actitud del profesorado. Cabe destacar como el profesorado con más experiencia no cambia, o lo hace muy poco, ante las propuestas formativas. La implicación del profesorado se hace imprescindible para cualquier cambio coincidiendo con Luz (2001) donde no es posible transformar la vida de quien no está comprometido con el cambio que se propone, de la misma manera que no es posible abrirse a la comunidad sin una participación activa en todos los sentidos (padres, sociedad, instituciones, etc.).

Algunas iniciativas con buenos resultados combinan la universidad con el ayuntamiento. Tomamos como ejemplo la iniciativa de la ciudad de Palencia que consiste en la creación de un seminario de formación que concluye en una escuela municipal de actividad física al aire libre 
(Miguel, 2006). Con esta premisa tenemos que, a nivel oficial, al seminario permanente se le concede mucha importancia, puesto que posee una potencialidad extraordinaria como contexto de interacción y de puesta en relación de la teoría y la práctica (Rodríguez, 1997), formato con el que estamos de acuerdo y permite un trabajo adecuado como así lo hemos destacado en el ejemplo anterior. Aunque en la actualidad con este formato de base podemos introducir algunos avances desde el punto de vista metodológico como veremos a continuación. La aplicación de las nuevas tendencias en la formación del profesorado nos ayudaría a resolver algunos de estos problemas, así lo plantea López (2004), creando un equipo de profesores que desde la propia experiencia puedan grupos de trabajos que conecten con las instituciones para avanzar en las nuevas líneas formativas. Ya desde la LOGSE, Gairín (1995) propone algunas propuestas de autores que se basan en el aprender a aprender y desarrollo organizado por uno mismo, con un proceso reflexivo sobre el propio aprendizaje.

Para Fernández (2006), la apuesta por una formación permanente eficaz es clave para facilitar el trabajo de las cuestiones planteadas anteriormente. Esta formación debe asentarse en dos pilares: 1.La experiencia docente. La experiencia diaria en las aulas genera la búsqueda de soluciones, el análisis sobre la propia práctica, desde los conocimientos psicológicos, sociológicos y pedagógicos, contribuye estructurar mejor las actividades y facilita la constante toma de decisiones en la práctica docente. 2.El centro educativo debe ser el eje. La experimentación curricular ayuda a romper con el individualismo tan frecuente y fomenta un trabajo colaborativo de búsqueda de soluciones comunes y de intercambio de todo tipo de experiencias. Entre otras actuaciones deseables tenemos la puesta en marcha de un buen programa de incentivos. Es necesario un reconocimiento real, por parte de las administraciones con competencias educativas hacia el profesorado implicado en proyectos interdisciplinares, intercentros, etc. Como aspecto positivo de todo el entramado formativo destacamos como Biscarri (1993) y Viciana y Salinas (2006) opina que el profesorado participa en la formación permanente independientemente del formato que se lleve a cabo, debido a factores intrínsecos como: que los programas sean aplicables en el aula, que aumenten el nivel de conocimiento en su materia y sea atractivo. La adaptación a los cambios y partiendo de una motivación para formarse nos parece de especial relevancia las aportaciones de Marcelo (1999) en cuanto que se empiezan a conocer estrategias concretas de desarrollo profesional que se aplican en función de las necesidades formativas y de la situación de los profesores. Aspectos de la formación permanente y las actividades al aire libre son tratados en la escuela francesa desde una perspectiva basada en una formación polivalente y práctica. Una actividad en la montaña permite al profesorado abordar la formación desde tres perspectivas: la práctica personal, la didáctica de la actividad y la puesta en práctica desde el punto de vista pedagógico (Allain, Claquin, y Ollier, 2005). Siendo el seminario permanente la opción frecuente en la formación del profesorado, el trabajo colaborativo es uno de sus pilares básicos. Éste nos permite afrontar y desarrollar proyectos con cierta solvencia, la construcción de una dinámica de trabajo cooperativo en los centros, que permita la propia autoevaluación, la formación desde la práctica de los profesores, y, de este modo, la mejora de aquellas facetas organizativas y pedagógicas de su trabajo que fueran definidas como susceptibles de mejora por los centros y los profesores (Escudero y Bolívar, 1994).

Si los cambios vienen desde las administraciones, es considerado de forma muy positiva por los entrevistados, pero no son menos importantes las últimas tendencias a nivel internacional recopiladas por Fernández (2006) como mantener la actualización pedagógica, dar sentido a la mejora del aprendizaje y las relaciones en el centro o apoyar un proyecto de reforma educativa. Aunque la formación tiende a un formato común para todo el profesorado, eliminando algunos formatos como los seminarios, charlas consensuadas con el profesorado...

Una vez visto los formatos formativos, los contenidos más destacados por los entrevistados son: los juegos de rastreo, senderismo, orientación e interpretación de mapas. Queda claro que la opción real es mucho más amplia como queda reflejado en la opinión de Vidal donde tienen cabida todos los contenidos de aire libre que puedan adaptarse al contexto escolar. Miguel (2006) sin nombrar ningún contenido de forma específica ofrece un trabajo interdisciplinar donde la educación ambiental es el eje de nuestra opción. El aula naturaleza nos permite acercarnos al medio natural mediante cualquier actividad físico-deportiva que vayamos a realizar en él. El descenso de un río en piragua 
requiere de una visión más globalizada de la actividad que el mero hecho de remar por un curso fluvial. Para Peñarrubia (2008) el profesorado de Educación Física incluye contenidos de actividades en la naturaleza que no están en el currículum como bicicleta todo terreno, escalada o barranquismo por su propio interés y gusto por este tipo de prácticas. El trabajo en la formación permanente nos permitiría un mayor y mejor conocimiento por parte del profesorado participante. González, Sánchez, Tabernero y Llanos (2004) nos hacen referencia al formato y contenidos principales en los cursos de formación. El 47,3\% versan sobre formación del currículo específica del área, el 27,7\% sobre aspectos evolutivos del alumnado en estas etapas y finalmente el $25 \%$ sobre aspectos didácticos como metodología, evaluación o recursos. Queremos resaltar que trabajan de forma muy general, sin profundizar en temas o contenidos concretos del área, quedándose fuera las actividades en el medio natural.

Y la tercera categoría, sobre el perfil ideal del profesorado a impartir contenidos de medio natural según la opinión de los entrevistados debe ser una combinación de profesorado en activo con experiencia en actividades en el medio natural y profesorado universitario que aseguren investigación e innovación. Deben tener una experiencia básica en actividades en el medio natural, así como una motivación e implicación para conseguir colaboración con otros centros e instituciones y la participación de expertos en contenidos específicos.

El profesorado con experiencia coincide con Humberstone (1993), que destaca cómo el profesorado opina que el contexto de la educación al aire libre es diferente al de la educación física escolar. Por lo tanto, hay que abordar la enseñanza con un enfoque no autoritario, de apoyo y centrado en el alumnado. Los modos de comunicación deben ser más simétricos y estimulantes. De esta manera se favorece la motivación y la confianza en sí mismo en el proceso de enseñanza-aprendizaje.

En la mayoría de las ocasiones es difícil separar la biografía de la persona con su pedagogía (Arribas, 2008; Fernández-Balboa, 1998). En nuestro caso, el profesorado implicado en la puesta en marcha de las actividades en la naturaleza tiene mucho de biografía vinculada a experiencias al aire libre. Sin embargo, destacan como la formación inicial recibida no está acorde con sus expectativas y en muchos casos se convierte en obstáculo para una adecuada implantación en el día a día como el resto de contenidos de Educación Física. Coincidiendo con Arribas (2008) y Fernández-Balboa y Muros (2004) nuestra posición frente a las actividades en el medio natural está condicionada por la formación recibida y los formadores que la llevaron a cabo. Estas premisas configuran un perfil con unas características muy concretas que tendrán que ir mejorando con otras actuaciones.

El presente trabajo, coincidiendo con Albarracín, Moreno y Beltrán (2014) nos ha permitido investigar sobre la situación actual de la Educación Física en los centros de secundaria andaluces y en particular sobre las actividades en el medio natural. Este trabajo puede suponer una importante contribución para la comunidad de científicos y profesionales educativos para justificar las bondades que se le atribuyen a las actividades al aire libre y sin embargo no se refleja en el trabajo diario dentro de las programaciones de la Educación Física (Arribas, 2008; Parra, 2001; Rousseau, 1969; SáezPadilla, 2010).

\section{Referencias}

Albarracín, A., Moreno, J. A. \& Beltrán, V. J. (2014). La situación actual de la educación física según el profesorado: un estudio cualitativo con profesores de la Región de Murcia. Cultura, Ciencia y Deporte, 27, 225-234.

Allain, J. L., Claquin, F., \& Ollier, B. (2005). Formar a los maestros para la polivalencia en las actividades en el medio natural: experiencias entre el IUFM y las escuelas primarias. En A. Miguel \& N. J. Bores (Eds.). III Congreso Internacional. El aula naturaleza en la Educación Física Escolar. Palencia: Asociación cultural cuerpo, educación y motricidad.

Anglada, P. (2008). Orígenes de los programas de aventura en la naturaleza con fines educativos. En L. Ariza \& M. Guillén (Eds.). IV Congreso Internacional y XXV Nacional de Educación Física. Córdoba: Universidad de Córdoba. 
Arribas, H. (2008). El pensamiento y la biografía del profesorado de Actividad Física en el Medio Natural: un estudio multicaso en la formación universitaria orientado a la comprensión de modelos formativos. Tesis doctoral inédita. Universidad de Valladolid.

Bacaicoa, F., \& Marín, M. (2001). La formación permanente del profesorado ¿para qué sirven los cursos de actualización? Revista Fuentes, 3, 32-44.

Biscarri, J. (1993). Motivaciones de los profesores respecto a su formación permanente. Revista interuniversitaria de formación del profesorado, 18, 221-237.

Cañadas, M., Ibáñez, S. J., García, J., Parejo, I., \& Feu, S. (2009). Estudio del conocimiento pedagógico de los entrenadores de baloncesto que trabajan etapas de formación. Habilidad Motriz. Revista de Ciencias de la Actividad Física y del Deporte, 32, 44-53.

Castillo, F., \& Almonacid, A. (2012). Las actividades en la naturaleza en la formación inicial docente: un acercamiento desde los sentidos. Educaçao e Pesquisa, 38, 667-681.

Escudero, J. M., \& Bolivar, A. (1994). Inovação e Formação Centrada na Escola. Uma Perspectiva da Realidade Espanhola. En A. Amiguihno \& R. Canário (Eds.). Escolas e Mudança: O Papel dos Centros de Formação. (pp. 97-155). Lisboa: Educa.

Fernández De Sanmamed, M. J. (2006). Diseño de estudios y diseños muestrales en investigación cualitativa. En M. L. Vázquez (Ed.). Introducción a las técnicas cualitativas de investigación aplicadas en salud. Cursos GRAAL 5. Colección Materiales 168. (pp. 31-51). Barcelona: Servicio de Publicaciones, Universidad Autónoma de Barcelona.

Fernández, F. L. (2006). La formación del profesorado. Revista LODE, 2, 4-5.

Fernández_Balboa, J. M. (1998). Transcending masculinities: Linking personhood and pedagogy. En C. Hickey, L. Fitzclarence \& R. Matthews (Eds.). Where the boys are. (pp.121-139). Geelong, Victoria: Deakin University Press.

Fullonet, F., \& Fuster, J. (2004). Actividades Físicas en la naturaleza. Tándem: Didáctica de la Educación Física, 16, 1-5.

Gairín, J. (1995). Estudio de las necesidades de formación de los equipos directivos de los centros educativos. Madrid: Centro de Publicaciones, MEC.

García Ruso, H. (1997). La formación del profesorado de Educación Física: problemas y expectativas. Barcelona: INDE.

García, S., \& Martínez, C. (2003). Enseñar a enseñar contenidos procedimentales es difícil. Revista Interuniversitaria de formación del profesorado, 17, 79-99.

Gómez Encinas, V. (2006). Juegos y actividades de reto y aventura en el contexto escolar. En J. M. Sáez, P. Sáenz-López \& M. Díaz (Eds.). Actividades en el medio natural. (pp. 9-22). Huelva: Servicio Publicaciones, Universidad de Huelva.

González, R. A., Sánchez, A., Tabernero, B., \& Llanos, C. (2004). Análisis de la formación permanente del profesorado de Educación Física a través de los C.P.R. y los C.F.I.E. en la provincia de Zamora. En actas del IV Congreso de la Asociación Española de Ciencias del Deporte. Valencia.

Granero, A., \& Baena, A. (2007). Importancia de los valores educativos de las actividades físicas en la naturaleza. Revista Habilidad Motriz, 29, 5-14.

Granero, A., \& Baena, A. (2011). Formación permanente del profesorado: juegos y deportes de aventura. Revista Internacional de Medicina y Ciencias de la Actividad Física y del Deporte, 11 (43), 531-547.

Higgins, P., \& Kirk, P. (2002). Teacher Education, Outdoor Education and Sustainability in Scotland. Planet, Special Edition Four, 8-12.

Higgins, P., Robbie, N., Beames, S., Cristhie, B., \& Scrutton, R. (2014). Education and culture committee. Outdoor Learning. The scottish parliament, 1-16.

Humberstone, B. (1993). El puesto y la importancia de la investigación etnográfica en el examen de aspectos relacionados con el género, la identidad y el poder en la educación física y al aire libre: ¿puede haber una etnografía feminista? Apuntes II Encuentro de Unisport de Sociología Deportiva. Málaga: Unisport.

Jaén, M., \& Banet, E. (2003). Formación inicial de profesores de secundaria: dificultades para aprender a planificar y desarrollar las actividades de enseñanza en aulas de secundaria. Revista Interuniversitaria de formación del profesorado, 17, 1, 51-78. 
López, F. L. (2004). Tendencias innovadoras en la formación del profesorado. Una propuesta de intervención: aprender a pensar desde el cuerpo. Revista Digital: Lecturas, Educación Física y Deportes, 77.

Lourenço, J. M. (2002). Vida en la Naturaleza. Revista Iberoamericana de Educación. Experiencias e Innovaciones. Educación Ambiental.

Louv, R. (2016). Vitamin N. 500 Ways to Enrich the Health \& Happiness of Your Family \& Community. Nort Carolina: Algonquin Books.

Luz, S. (2001). El Baúl de los Recursos: proyecto para la organización, adquisición y optimización de materiales para el aprendizaje. Revista iberoamericana de educación. Experiencias e innovaciones. Recursos.

Marcelo, C. (1999). Formación del profesorado para el cambio educativo. Barcelona: Ediciones Universitarias de Barcelona, S.L.

Martínez Mediano, C. (2004). Técnicas e instrumentos de recogida y análisis de datos. Madrid: UNED.

Maxwell, J. A. (2010). Using Numbers in Qualitative Research. Qualitative Inquiry, 16, 475-482.

Mellado, A., \& Mellado, J. L. (2006). El campamento como medio educativo. Revista de Estudios de Juventud: Jóvenes y campamentos de verano, 72, 25-36.

Miguel, A. (2006). La escuela municipal de actividades físicas en el medio natural: un modelo de formación. En J. M. Sáez, P. Sáenz-López \& M. Díaz (Eds.). Actividades en el medio natural. (pp. 83-96). Huelva: Servicio Publicaciones, Universidad de Huelva.

Mogollón, A. S., \& Vázquez, M. L. (2006). Técnicas cualitativas aplicadas en salud. En M. L. Vázquez (Ed..). Introducción a las técnicas cualitativas de investigación aplicadas en salud. Cursos GRAAL 5. Colección Materiales 168. (pp. 53-81). Barcelona: Servicio de Publicaciones, Universidad Autónoma de Barcelona.

Napper-Owen, G. E., Kovar, S. K., Ermler, K. L., \& Mehrhof, J. H. (1999). Curricula equity in required ninthgrade physical education. Journal of teaching in physical education, 19, 2-21.

Nasser, D. (1994). Pedagogía de la aventura: ¿Una necesidad en la sociedad moderna? Apuntes I Curso de Especialización “Ocio y Recreación deportiva”. Madrid: INEF, Universidad Politécnica de Madrid.

Orden de 9 de junio de 2003, por la que se aprueba el II Plan Andaluz de Formación Permanente del Profesorado.

Osses, S., Sánchez, I., \& Ibáñez, F. M. (2006). Investigación en Educación. Hacia la generación de teoría a través del proceso analítico. Revista Estudios Pedagógicos, 32, 119-133.

Parra, M. (2001). Programa de actividades físicas en la naturaleza y deportes de aventura para la formación del profesorado de segundo ciclo de Secundaria. Tesis doctoral inédita. Universidad de Granada.

Parra, M. (2006). La acampada como medio educativo en primaria y secundaria. En J. M. Sáez, P. Sáenz-López \& M. Díaz (Eds.). Actividades en el medio natural. (pp. 23-36). Huelva: Servicio Publicaciones, Universidad de Huelva.

Parra, M., Rovira, C., \& Luque, P. (2002). Aproximación taxonómica de las actividades físicas en la naturaleza en los centros educativos. Revista Retos: Nuevas Tendencias en Educación Física, Deporte y Recreación, 2, 6-14.

Peñarrubia, C. (2008). Las actividades físico-deportivas en el medio natural. Estado de la situación actual en la ciudad de Zaragoza. Etapa de Secundaria. En actas del V Congreso de la Asociación Española de Ciencias del Deporte. León.

Pereira, A. L., \& Félix, M. J. (2002). Siglo XXI: nuevos valores, nuevas profesiones. Una perspectiva del ocio deportivo en la naturaleza integrado en el turismo. Revista Digital: Lecturas, Educación Física y Deportes, 50.

Pila Teleña, A. (1981). Educación físico-deportiva: fundamentos generales del programa. Madrid: Editorial Pila Teleña.

Rivera, M. (2010). Turismo activo en la naturaleza y espacios de ocio en Andalucía: aspectos territoriales, políticas públicas y estrategias de planificación. Sevilla: Consejería Turismo, Comercio y Deporte.

Rodríguez López, J. M. (1995). Formación de profesores y prácticas de enseñanza. Huelva: Servicio de Publicaciones, Universidad de Huelva.

Rodríguez López, J. M. (1997). Bases y estrategias de formación permanente. Huelva: Hergué Impresores S. L.

Rousseau, J. J. (1969). Emilio o de la Educación. Madrid: Edaf. 
Sáez-Padilla, J. (2010). La formación del profesorado de Educación Física de ESO en Andalucía en relación con las Actividades en el Medio Natural. Huelva: Servicio de Publicaciones, Universidad de Huelva.

Sáez-Padilla, J., \& Fuentesal, J. (2014). La formación universitaria en actividades físico-deportivas en el medio natural: situación actual y perspectivas de futuro. Tándem: Didáctica de la Educación Física, 45, 14-21.

Salinas, F. (2006). La planificación de los bloques contenidos de Educación Física en Educación Secundaria Obligatoria. Revista Digital: CienciayDeporte.net, 3.

Sánchez, M. (2014). Educar en contacto con la naturaleza: Noruega como referente. Trabajo fin de grado. Valladolid: Universidad de Valladolid.

Santos, M. L. (2003). Las actividades en el medio natural en la Educación Física Escolar. Sevilla: Wanceulen.

Santos, M. L., \& Muñoz, F. (2006). Las actividades en el medio natural en la escuela. Consideraciones para un tratamiento educativo. En J. M. Sáez, P. Sáenz-López \& M. Díaz (Eds.). Actividades en el medio natural. (pp. 37-64). Huelva: Servicio Publicaciones, Universidad de Huelva.

Santos, M. L., \& Muñoz, F. (2007). El medio natural como eje pedagógico. El sentido educativo de las actividades físico-deportivas en el medio natural escolar. En Actas V Congreso Internacional, el aula naturaleza en la Educación Física Escolar. Palencia.

Stake, R. E. (2006). Multiple Case Study Analysis. New York. London: The Guilford press.

Vázquez, M. L. (Ed.) (2006). Introducción a las técnicas cualitativas de investigación aplicadas en salud. Cursos GRAAL 5. Colección Materiales 168. Barcelona: Servicio de Publicaciones, Universidad Autónoma de Barcelona.

Vegas, E. (2006). Incentivos docentes y sus efectos en el aprendizaje de Latinoamérica. Revista Educación, 340, 213-242.

VICIANA, J., \& Salinas, F. (2006). La formación permanente del profesorado de Educación Física. Un campo habitado en los últimos años. Revista Digital: Lecturas, Educación Física y Deportes, 103. 